2. To: (Receiving Organization)

FDH 324 RIVER CORRIDOR PROJECT

5. Proj./Prog./Dept./Dlv.:

BUILDING 324 B-CELL CLEAN-CUT

3. From: (Originating Organization)
WASTE MANAGEMENT TECHNICAL SERVICE
6. Design Authority/Design Agent/Cog. Engr.:
WM BOEHNKE/BD FLANAGAN/WM BOEHNKE

8: Originator Remarks:

RELEASE OF THE 22 TON STEEL WASTE DISPOSAL BOX LIFTING BAIL DESIGN CALCULATIONS AND RELATED DESIGN DATA.

15.

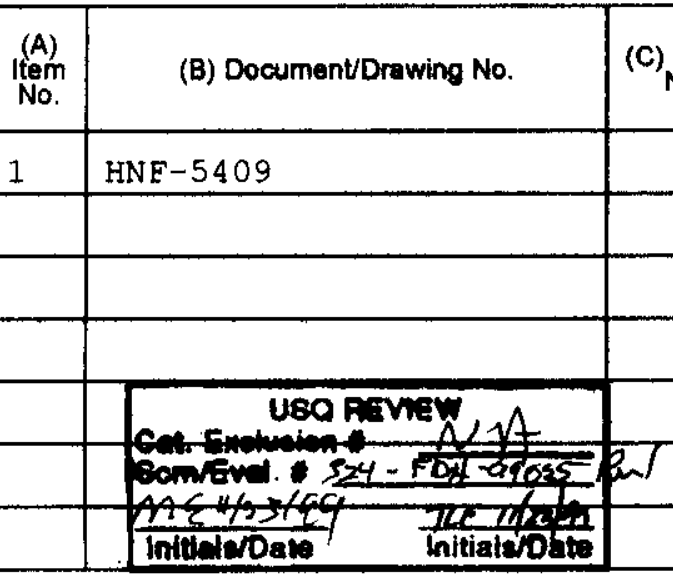

16.

\begin{tabular}{|c|l|}
\hline Approval Dealgnator (F) & \\
\hline E, S, Q, D OR N/A & 1. Approval \\
(See WHC.CM-3-5, & 2. Rolease \\
Sec. 12.7) & 3. Information \\
\hline
\end{tabular}

17.

\begin{tabular}{|c|c|c|c|}
\hline $\begin{array}{l}\text { (G) } \\
\text { Rea- } \\
\text { son. }\end{array}$ & $\begin{array}{l}(\mathrm{H}) \\
\text { Disp. }\end{array}$ & (J) Name & ( \\
\hline 2 & 1 & Dwelon Autl & nority WM \\
\hline 2 & 1 & Design Age & nt $B D \quad F I$ \\
\hline 2 & 1 & Cog. Eng. & WM BOEH \\
\hline 2 & 1 & Cog. Mgr. & JA REEV \\
\hline 2 & 1 & QA & JA CHAF \\
\hline \multirow[t]{2}{*}{2} & 1 & Safety & T ROMAN \\
\hline & & Env. & $\mathrm{NA}$ DOE \\
\hline $\mathrm{WM}^{\mathrm{C}}$ & & & Illapp \\
\hline $\begin{array}{l}\text { Sign } \\
\text { Orig }\end{array}$ & reo 0 & $\overline{\mathrm{DT}}$ & Dato \\
\hline
\end{tabular}

Reason for Transmittal (G)

4. Review

5. Post-Review

6. Dist. (Receipt Acknow. Requlred)

KEY

$\mathrm{n} 22 / \mathrm{r}_{\mathrm{H} 1-11}$
11A. Design Baseline Document? $O$ Yes $\bigcirc$ No

\begin{tabular}{|c|}
\hline $\begin{array}{l}\text { 4. Related EDT No:: } \\
\text { NA }\end{array}$ \\
\hline $\begin{array}{l}\text { 7. Purchase Order No: } \\
\text { NA }\end{array}$ \\
\hline $\begin{array}{l}\text { 9. Equip./Component No.: } \\
\text { NA }\end{array}$ \\
\hline $\begin{array}{l}\text { 10. System/Bido./Facility: } \\
324 / \text { REC/B-CELL }\end{array}$ \\
\hline $\begin{array}{l}\text { 12. Major Assm. Dwg. No.: } \\
\text { H-3-56431 }\end{array}$ \\
\hline $\begin{array}{l}\text { 13. Permit/Permit Application No.: } \\
\text { NA }\end{array}$ \\
\hline $\begin{array}{l}\text { 14. Required Response Date: } \\
\text { NA }\end{array}$ \\
\hline
\end{tabular}

\begin{tabular}{l} 
4. Related EDT No.: \\
NA \\
7. Purchase Order No.: \\
NA \\
9. Equip./Component No.: \\
NA \\
10. System/Bidg./Facility: \\
324/REC/B-CELL \\
12. Major Assm. Dwg. No.: \\
H-3-564 31 \\
13. Permit/Permit Application No.: \\
NA \\
\hline 14. Required Response Date: \\
NA
\end{tabular}

(F)

(G)

(E) Title or Description of Data Transmitted

A

\begin{tabular}{c|c|c|c}
$\begin{array}{c}\text { Approval } \\
\text { Desig- } \\
\text { nator }\end{array}$ & $\begin{array}{c}\text { Reason } \\
\text { for Trans- } \\
\text { mittal }\end{array}$ & $\begin{array}{c}\text { Origl- } \\
\text { nator } \\
\text { Dispo- } \\
\text { stion }\end{array}$ & $\begin{array}{c}\text { Receiv- } \\
\text { er } \\
\text { Dispo- } \\
\text { sition }\end{array}$ \\
\hline
\end{tabular}

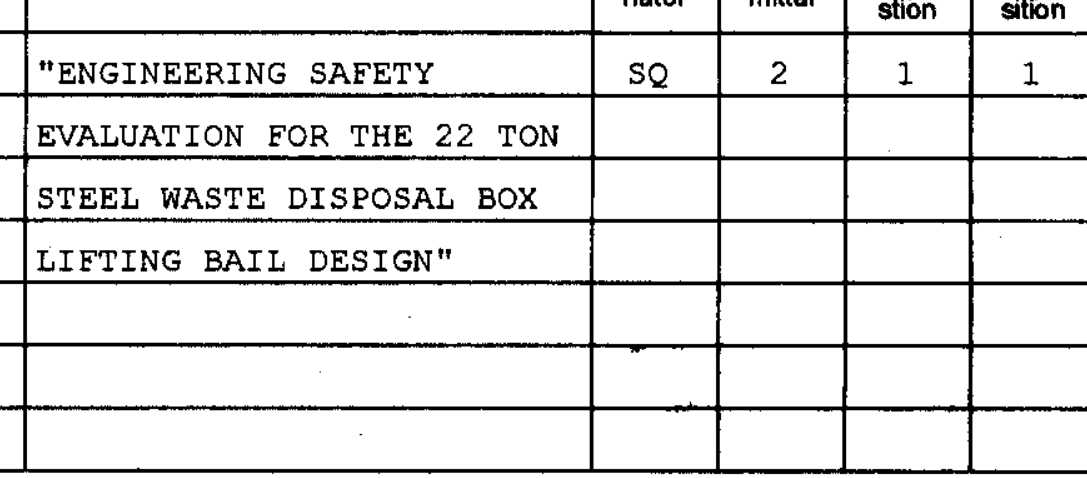
SIGNATURE/DISTRIBUTION (See Approval Designator for requlred signatures)

\begin{tabular}{lll|l|l|l} 
(K) Signature & (L) Date & (M) MSIN & Rea- & (H) & (J) Name
\end{tabular}

(K) Signature

(L) Date

(M) MSIN

INDEPENDENT REVIEWER

DI RILEY DJ/e ter DaRiay $1 / 14 / 99 \mathrm{H} 1-11$

sen

1

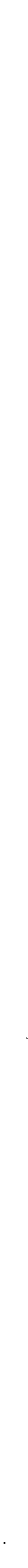




\section{ENGINEERING SAFETY EVALUATION FOR 22 TON STEEL DISPOSAL BOX LIFTING BAIL DESIGN}

WM BOFHINKE

WASTE MANAGEMENT TECHNICAL SERVICES

Richland, WA 99352

U.S. Department of Energy Contract DE-AC06-96RL13200

EDT/ECN: EDT 628121

UC: 2000

Ong Code: SR550000

B\&R Code: EW04J1070

Charge Code: '101047/B000 KN HMN400/l
Total Pages:
21

Key Words:

Analysis on the modified lifting bails for the 22 Ton steel waste Disposal Box.

Abstract:

The objective of this analysis is to design and analyze the lifting bail of the 22 Ton steel Waste Disposal Box (SWDB). The new design takes the original lifting bail and adds a hinge allowing the top portion of the bail to fold over towards the lid. This modification only applies to the lifting bails and does not effect the attachment of the bails to the box, this analysis applies only to the lifting bails hinge design.

TRADEMARK DISCLAIMER. Reference herein to any specific commercial product, process, or service by trade name, trademark, manufacturer, or otherwise, does not necessarlly constitute or imply its endorsement, recommendation, or favoring by the United States Government or any agency thereof or its contractors or subcontractors.

Printed in the United States of America. To obtain copies of this document, contact: Document Control Services, P.O. Box 950, Maitstop H6-08, Richland WA 99352, Phone (509) 372-2420; Fax (509) 376-4989.

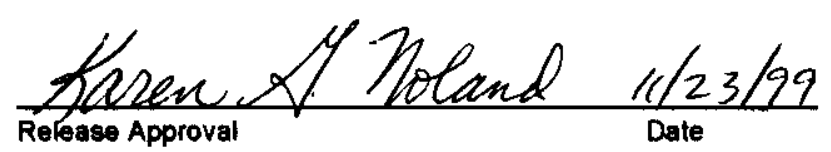

Approved For Public Release 


\section{ENGINEERING SAFETY EVALUATION}

Subject: 22 Ton Steel Waste Disposal Box Lifting Bail Design

Preparer:

Checker:

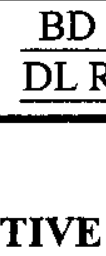

\subsection{OBJECTIVE}

The objective of this analysis is to design and analyze the lifting bail of the 22 Ton Steel Waste Disposal Box. The new design takes the original lifting bail and adds a hinge allowing the bail to fold over towards the lid. A spring is used to hold the top of the bail at a 52 degree angle so the bail can be remotely pushed to the vertical position. Since the new design does not change the attachment to the box or the top of the bail which interfaces with the original lifting yoke, this analysis is limited to the design of the hinge.

The lifting bails must withstand a factor of safety for lifting of 3:1 to yield.

\subsection{REFERENCES}

AISC, 1989, Manual of Steel Construction, Allowable Stress Design, $9^{\text {th }}$ Edition, American Institute of Steel Construction, Inc., Chicago, Illinois.

PNL, 1988, 22 Ton Steel Waste Disposal Box Arrangement, drawing H-3-56431, Rev. 1, Pacific Northwest Laboratory, Richland, Washington.

Shigley, Joseph E, and Charles R. Mischke, 1989, Mechanical Engineering Design, Fifth Edition, McGraw-Hill, Inc. New York, New York.

DWS, 1999, Lifting Bail Spring, (Fax to D. Reid, 9/23/99, 13:29), 800-238-3510, Diamond Wire Spring Co., Tyler, Texas.

\subsection{ASSUMPTIONS, RESULTS, AND CONCLUSIONS}

The lifting bail design consists of three main components. The components are the top and bottom parts of the bail, the hinge pin, and the spring. The maximum weight of the package that is used in the calculations is conservatively estimated to be $30,000 \mathrm{lb}$ on each lifting bail $(60,000 \mathrm{lb}$ total). The margin of safety for each evaluation includes a lifting factor of $3: 1$ to yield. 


\section{ENGINEERING SAFETY EVALUATION}

Subject: 22 Ton Steel Waste Disposal Box Lifting Bail Design

Preparer: BD Flanagan IEDF

Checker: DL Riley $Q_{4 B}$ for

Page: 2 of $\underline{20}$

Date: $\overline{9 / 22} / 99$

Date: $9 / 22 / 99$

The hinge pin is made of AISI 41L40 with a yield stress of 120,000 psi. A two step process was used to determine the bending of the pin for this design. The first step used the formulas for continuous beams, with the theory of three moments. The second step utilizes the simple beam formulas for a uniformly distributed load and variable end moments. The end moments are taken from the moments determined from the first step and added in the second step to find the maximum moment for each span. The moments are symmetric about the center so only one side of the maximum moments are determined. The maximum moment is found on the forth span to be $2,151 \mathrm{in}-\mathrm{lb}$. The maximum bending stress in the pin is thus 32,706 psi which gives a margin of safety of 0.22 . The largest shear stress of the pin is 6,603 psi with a margin of safety of 2.03 . The largest bending stress at the reaction points is 9,032 psi with a margin of safety of 3.43 . From the bending and shear stresses the principle stresses are determined and are shown to be 9,871 psi and 6,603 psi for shear. The margin of safety is 3.05 and 0.22 respectively. The bearing stress of the pin is 4,898 psi with a margin of safety of 3.08 .

The top and bottom bails were evaluated for direct tensile stress, shear tear-out stress, and bearing stress using AISI 1040 with a yield stress of 42,000 psi. Both the top and bottom bails have the same area for hinge teeth. Therefore, stresses will be the same for both top and bottom bails. The direct tensile stress is 3,820 psi with a margin of safety of 2.67 . The shear tear-out stress is 4,193 psi with a margin of safety of 0.67 . The bearing stress is 4,881 psi with a margin of safety of 1.87 .

The maximum moment required to lift the top bail by the spring was calculated to be $825 \mathrm{in}-\mathrm{lb}$ about the center of the pin. At 60 degrees the moment required is $413 \mathrm{in}-\mathrm{lb}$. Spring $\phi 2.0 \times 3.86 \times \phi .37417-7$ by Diamond Wire Spring Co. was determined to sufficiently provide the required moments and allows the lifting bail to be positioned at a steady state angle of 52 degrees. 


\section{ENGINEERING SAFETY EVALUATION}

Subject: 22 Ton Steel Waste Disposal Box Lifting Bail Design

Preparer: BD Flanagan

Checker: DL Riley Saß for
4.0 EVALUATION
Hinge Design
(H-3-56431)

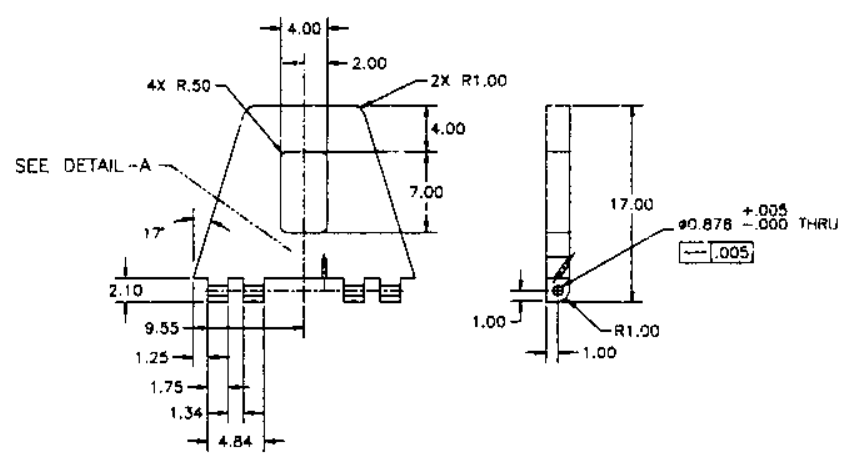

Page: 3 of $\underline{20}$

Date: $\overline{9 / 2} 2 / 99$

Date: $9 / 22 / 99$

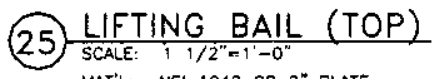

MAT'L: AISI 1040 CS $2^{*}$ FLATE

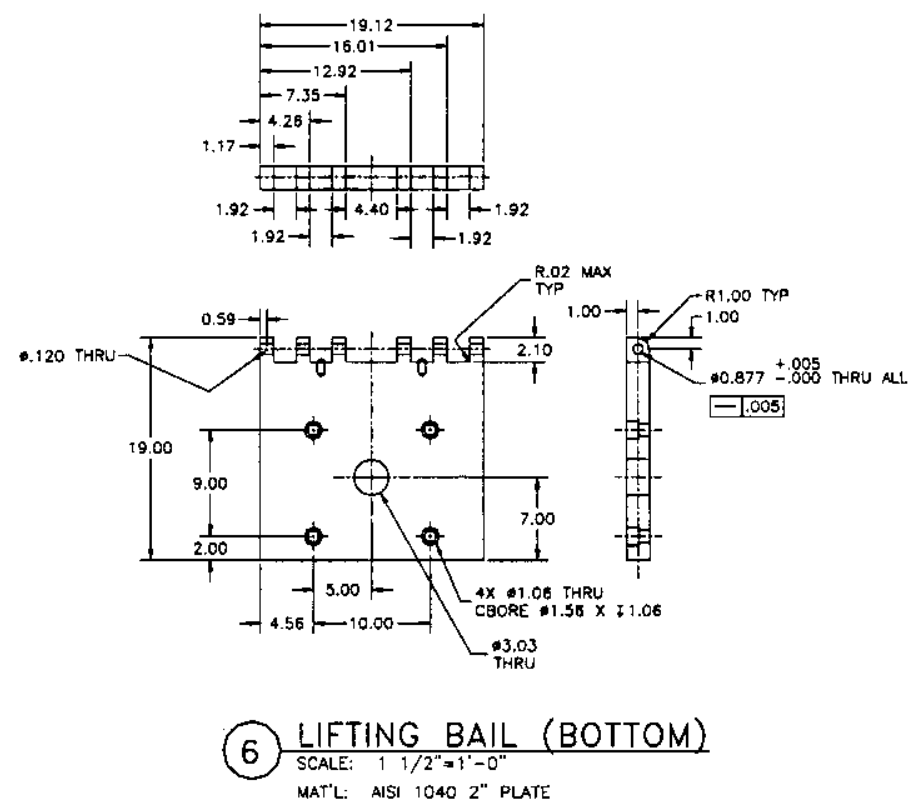

WMNW-001 3/99 


\section{ENGINEERING SAFETY EVALUATION}

Subject: 22 Ton Steel Waste Disposal Box Lifting Bail Design

Preparer: BD Flanagan TEF

Page: 4 of $\underline{20}$

Date: $9 / 22 / 99$

Checker: DL Riley $Q \omega B$ for

\section{Evaluation of Pin}

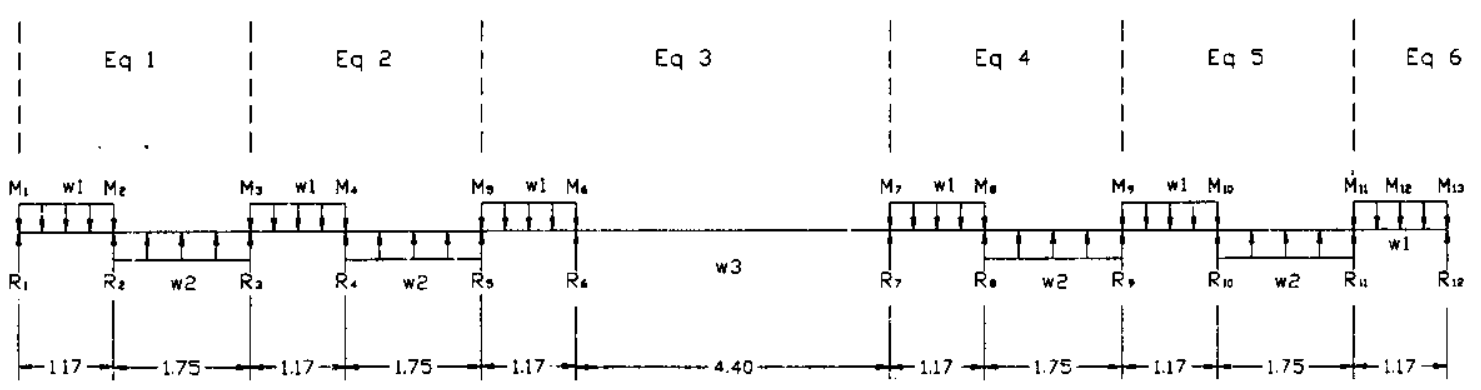

Using the equation for the theorem of three moments (AISC 1989, 2-294):

$M_{1} L_{1}+2 M_{2}\left(L_{1}+L_{2}\right)+M_{3} L_{2}+\frac{w L_{1}^{3}}{4}+\frac{w L_{2}^{3}}{4}$

Assume there are no gaps between the hinge teeth.

$F:=30000$

$\mathrm{L}_{1}:=1.17 \quad \mathrm{~L}_{2}:=1.75 \quad \mathrm{~L}_{3}:=4.4$

$w_{1}:=\frac{F}{6 \cdot 1.17} \quad w_{2}:=\frac{-F}{4 \cdot 1.75}$

$\mathrm{w}_{1}=4273.5 \quad \mathrm{w}_{2}=-4285.71$

$w_{3}:=0$

$\mathrm{M}_{1}:=0$ 


\section{ENGINEERING SAFETY EVALUATION}

Subject: 22 Ton Steel Waste Disposal Box Lifting Bail Design

Preparer: BD Flanagan TBDF

Checker: DL Riley 2 WB fer

Page: 5 of 20

Date: $\overline{9 / 22} / 99$

Date: $9 / 22 / 99$

\begin{tabular}{|c|c|c|c|}
\hline \multirow{6}{*}{$\begin{array}{l}\text { Guess values } \\
\text { for Mathcad: }\end{array}$} & $\mathrm{M}_{1}:=0$ & $M_{7}:=-150$ & $\mathrm{M}_{13}:=0$ \\
\hline & $\mathrm{M}_{2}:=500$ & $\mathrm{M}_{8}:=600$ & \\
\hline & $M_{3}:=450$ & $M_{9}:=450$ & \\
\hline & $M_{4}:=450$ & $M_{10}:=450$ & \\
\hline & $M_{5}:=600$ & $M_{11}:=500$ & \\
\hline & $M_{6}:=-150$ & $M_{12}:=200$ & \\
\hline
\end{tabular}

Given due to symmetry

$\begin{array}{ll}M_{2}=M_{11} & M_{4}=M_{9} \\ M_{3}=M_{10} & M_{5}=M_{8}\end{array}$

Equation 1

$$
\mathrm{M}_{1} \cdot \mathrm{L}_{1}+2 \cdot \mathrm{M}_{2} \cdot\left(\mathrm{L}_{1}+\mathrm{L}_{2}\right)+\mathrm{M}_{3} \cdot \mathrm{L}_{2}+\frac{\mathrm{w}_{1} \cdot \mathrm{L}_{1}^{3}}{4}+\frac{\mathrm{w}_{2} \cdot \mathrm{L}_{2}^{3}}{4}=0
$$

Equation 2

$$
\mathrm{M}_{3} \cdot \mathrm{L}_{1}+2 \cdot \mathrm{M}_{4} \cdot\left(\mathrm{L}_{1}+\mathrm{L}_{2}\right)+\mathrm{M}_{5} \cdot \mathrm{L}_{2}+\frac{\mathrm{w}_{1} \cdot \mathrm{L}_{1}^{3}}{4}+\frac{\mathrm{w}_{2} \cdot \mathrm{L}_{2}{ }^{3}}{4}=0
$$

Equation 3

$$
\mathrm{M}_{5} \cdot \mathrm{L}_{1}+2 \cdot \mathrm{M}_{6} \cdot\left(\mathrm{L}_{1}+\mathrm{L}_{3}\right)+\mathrm{M}_{7} \cdot \mathrm{L}_{3}+\frac{\mathrm{w}_{1} \cdot \mathrm{L}_{1}{ }^{3}}{4}+\frac{\mathrm{w}_{3} \cdot \mathrm{L}_{3}{ }^{3}}{4}=0
$$

Equation 4

$$
\mathrm{M}_{7} \cdot \mathrm{L}_{1}+2 \cdot \mathrm{M}_{8} \cdot\left(\mathrm{L}_{1}+\mathrm{L}_{2}\right)+\mathrm{M}_{9} \cdot \mathrm{L}_{2}+\frac{\mathrm{w}_{1} \cdot \mathrm{L}_{1}{ }^{3}}{4}+\frac{\mathrm{w}_{2} \cdot \mathrm{L}_{2}{ }^{3}}{4}=0
$$




\section{ENGINEERING SAFETY EVALUATION}

Subject: 22 Ton Steel Waste Disposal Box Lifting Bail Design

Preparer: BD Flanagan

Checker: DL Riley OAB for

Page: 6 of 20

Date: $9 / 22 / 99$

Date: $9 / 22 / 99$

Equation 5

$$
M_{9} \cdot L_{1}+2 \cdot M_{10} \cdot\left(L_{1}+L_{2}\right)+M_{11} \cdot L_{2}+\frac{w_{1} \cdot L_{1}{ }^{3}}{4}+\frac{w_{2} \cdot L_{2}{ }^{3}}{4}=0
$$

Equation 6

$$
M_{12}-w_{1} \cdot \frac{L_{1}^{2}}{8}+\frac{M_{11}+M_{13}}{2}-\frac{\left(M_{11}-M_{13}\right)^{2}}{2 \cdot w_{1} \cdot L_{1}^{2}}=0
$$

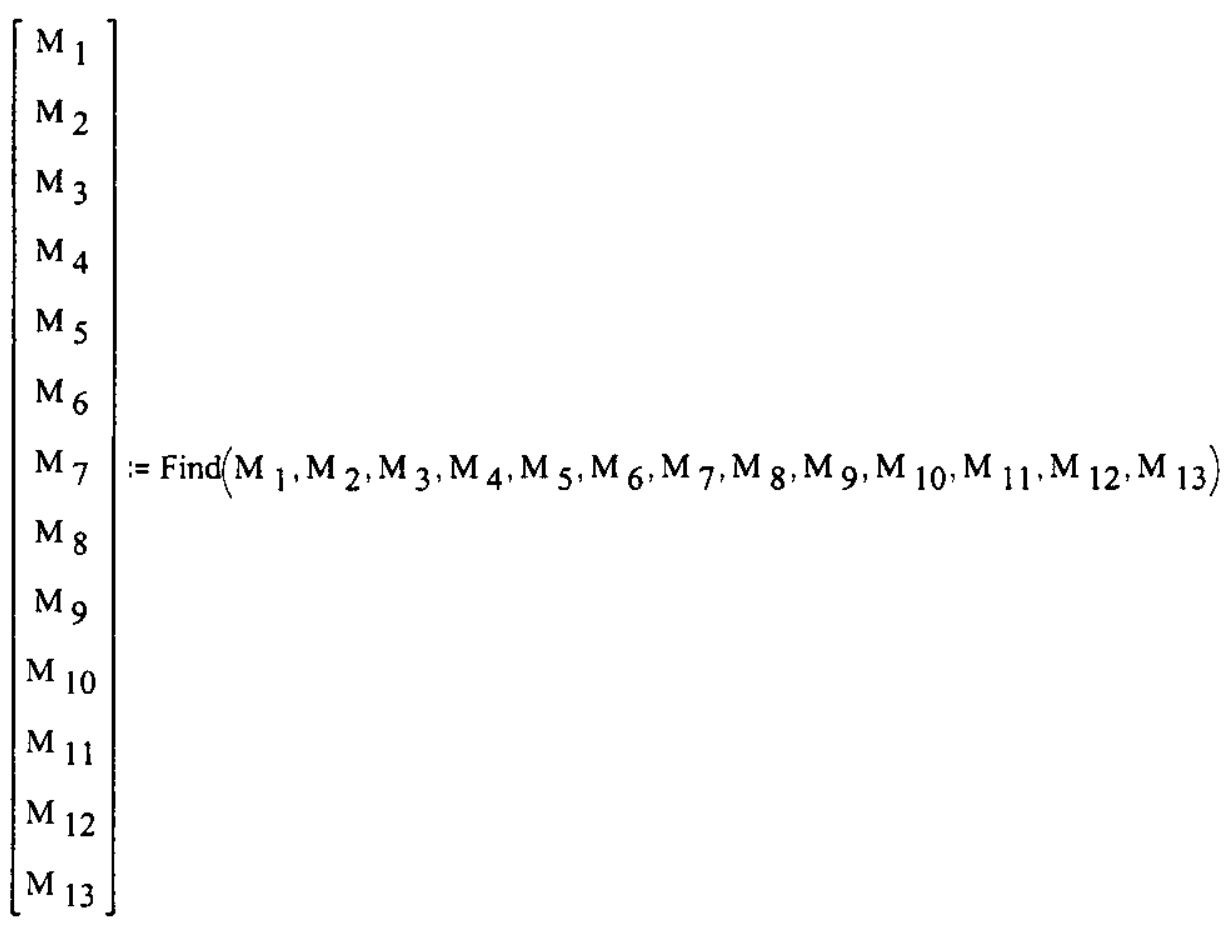




\section{ENGINEERING SAFETY EVALUATION}

Subject: 22 Ton Steel Waste Disposal Box Lifting Bail Design

Preparer: BD Flanagan

Checker: DL Riley Daß for

Page: 7 of 20

Date: $\overline{9 / 2} 2 / 99$

Date: $9 / 22 / 99$

Results:

$\begin{array}{lll}M_{1}=0 & M_{6}=-154.84 & M_{11}=559.1 \\ M_{2}=559.1 & M_{7}=-154.84 & M_{12}=478.4 \\ M_{3}=437.65 & M_{8}=594.05 & M_{13}=0.02 \\ M_{4}=424.56 & M_{9}=424.56 & \\ M_{5}=594.05 & M_{10}=437.65 & \end{array}$

Largest moment is $\mathrm{M}_{5}=\mathrm{M}_{8}=594.05$ in-lb.

Find maximum moment between spans

Span 1

$$
\begin{aligned}
& M_{\max 1}:=\frac{w_{1} \cdot L_{1}^{2}}{8}-\frac{M_{1}+M_{2}}{2}+\frac{\left(M_{1}-M_{2}\right)^{2}}{2 \cdot w_{1} \cdot L_{1}{ }^{2}} \\
& M_{\max 1}=478.42
\end{aligned}
$$

Span 2

$$
\begin{aligned}
& M_{\max 2}:=\frac{w_{2} \cdot L_{2}{ }^{2}}{8}-\frac{M_{2}+M_{3}}{2}+\frac{\left(M_{2}-M_{3}\right)^{2}}{2 \cdot w_{2} \cdot L_{2}{ }^{2}} \\
& M_{\max 2}=-2139.57
\end{aligned}
$$

Span 3

$$
\begin{aligned}
& M_{\max 3}:=\frac{w_{1} \cdot L_{1}{ }^{2}}{8}-\frac{M_{3}+M_{4}}{2}+\frac{\left(M_{3}-M_{4}\right)^{2}}{2 \cdot w_{1} \cdot L_{1}{ }^{2}} \\
& M_{\max 3}=300.16
\end{aligned}
$$

WMNW-0013/99 


\section{ENGINEERING SAFETY EVALUATION}

Subject: 22 Ton Steel Waste Disposal Box Lifting Bail Design

Preparer: BD Flanagan Tito

Checker: DL Riley Dafs for

Page: 8 of 20

Date: $\overline{9 / 2} 2 / 99$

Date: $9 / 22 / 99$

Span 4

$$
\begin{aligned}
& M_{\max 4}:=\frac{w_{2} \cdot L_{2}{ }^{2}}{8}-\frac{M_{4}+M_{5}}{2}+\frac{\left(M_{4}-M_{5}\right)^{2}}{2 \cdot w_{2} \cdot L_{2}{ }^{2}} \\
& M_{\max 4}=-2151.02
\end{aligned}
$$

Span 5

$$
\begin{aligned}
& M_{\max 5}:=\frac{w_{1} \cdot L_{1}{ }^{2}}{8}-\frac{M_{5}+M_{6}}{2}+\frac{\left(M_{5}-M_{6}\right)^{2}}{2 \cdot w_{1} \cdot L_{1}{ }^{2}} \\
& M_{\max 5}=559.58
\end{aligned}
$$

Span 6

$$
\begin{aligned}
& M_{\max 6}:=\frac{w_{3} \cdot L_{3}{ }^{2}}{8}-\frac{M_{6}+M_{7}}{2} \\
& M_{\max 6}=154.84
\end{aligned}
$$

Maximum moment is $\mathrm{M}_{\max 4}=-2151.02 \mathrm{in}-\mathrm{lb}$.

Find reaction forces to determine shear:

Span 1

$$
\begin{array}{ll}
\mathrm{R}_{1}:=\frac{\mathrm{w}_{1} \cdot \mathrm{L}_{1}}{2}-\frac{\mathrm{M}_{1}-\mathrm{M}_{2}}{\mathrm{~L}_{1}} & \mathrm{R}_{2 \mathrm{a}}:=\frac{\mathrm{w}_{1} \mathrm{~L}_{1}}{2}+\frac{\mathrm{M}_{1}-\mathrm{M}_{2}}{\mathrm{~L}_{1}} \\
\mathrm{R}_{1}=2977.87 & \mathrm{R}_{2 \mathrm{a}}=2022.13
\end{array}
$$




\section{ENGINEERING SAFETY EVALUATION}

Subject: 22 Ton Steel Waste Disposal Box Lifting Bail Design

Preparer:

Checker:

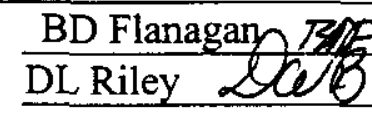

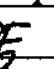

Page: 9 of 20

Date: $9 / 22 / 99$

Date: $9 / 22 / 99$

Span 2

$$
\begin{array}{ll}
\mathrm{R}_{2 \mathrm{~b}}:=\frac{\mathrm{w}_{2} \cdot \mathrm{L}_{2}}{2}+\frac{\mathrm{M}_{2}-\mathrm{M}_{3}}{\mathrm{~L}_{2}} & \mathrm{R}_{3 \mathrm{a}}:=\frac{\mathrm{w}_{2} \cdot \mathrm{L}_{2}}{2}-\frac{\mathrm{M}_{2}-\mathrm{M}_{3}}{\mathrm{~L}_{2}} \\
\mathrm{R}_{2 \mathrm{~b}}=-3680.6 & \mathrm{R}_{3 \mathrm{a}}=-3819.4
\end{array}
$$

Span 3

$$
\begin{array}{ll}
\mathrm{R}_{3 b}:=\frac{w_{1} \cdot L_{1}}{2}+\frac{M_{3}-M_{4}}{L_{1}} & R_{4 a}:=\frac{w_{1} \cdot L_{1}}{2}-\frac{M_{3}-M_{4}}{L_{1}} \\
R_{3 b}=2511.19 & R_{4 a}=2488.81
\end{array}
$$

Span 4

$$
\begin{array}{lc}
\mathrm{R}_{4 b}:=\frac{\mathrm{w}_{2} \cdot \mathrm{L}_{2}}{2}-\frac{\mathrm{M}_{4}-\mathrm{M}_{5}}{\mathrm{~L}_{2}} & \mathrm{R}_{5 \mathrm{a}}:=\frac{\mathrm{w}_{2} \cdot \mathrm{L}_{2}}{2}+\frac{\mathrm{M}_{4}-\mathrm{M}_{5}}{\mathrm{~L}_{2}} \\
\mathrm{R}_{4 \mathrm{~b}}=-3653.15 & \mathrm{R}_{5 \mathrm{a}}=-3846.85
\end{array}
$$

Span 5

$$
\begin{array}{ll}
R_{5 b}:=\frac{w_{1} L_{1}}{2}+\frac{M_{5}-M_{6}}{L_{1}} & R_{6 a}:=\frac{w_{1} \cdot L_{1}}{2}-\frac{M_{5}-M_{6}}{L_{1}} \\
R_{5 b}=3140.07 & R_{6 a}=1859.93
\end{array}
$$

Span 6

$$
\begin{array}{ll}
R_{6 b}:=\frac{M_{6}-M_{7}}{L_{3}} & R_{7 a}:=\frac{M_{6}-M_{7}}{L_{3}} \\
R_{6 b}=0 & R_{7 a}=0
\end{array}
$$




\section{ENGINEERING SAFETY EVALUATION}

Subject: 22 Ton Steel Waste Disposal Box Lifting Bail Design

Preparer: BD Flanagan BE

Checker: DL Riley oleobi for

Page: 10 of 20

Date: $\overline{9 / 2} 2 / 99$

Date: $9 / 22 / 99$

Add reactions together for each span

$$
\begin{array}{ll}
R_{2}:=R_{2 a}+R_{2 b} & R_{4}:=R_{4 a}+R_{4 b} \quad R_{6}:=R_{6 a}+R_{6 b} \\
R_{3}:=R_{3 a}+R_{3 b} & R_{5}:=R_{5 a}+R_{5 b} \\
R_{1}=2977.87 & \\
R_{2}=-1658.47 & \\
R_{3}=-1308.21 & \\
R_{4}=-1164.34 \\
R_{5}=-706.78 \\
R_{6}=1859.93
\end{array}
$$

Maximum reaction force is $R_{1}=2977.87 \mathrm{lbf}$.

Determine Shear Stresses at reaction points from the beam:

$$
\begin{aligned}
& \mathrm{A}_{\text {pin }}:=\frac{\pi}{4} \cdot 875^{2} \quad \mathrm{~A}_{\text {pin }}=0.601 \mathrm{in}^{2} \\
& { }^{\tau_{1}}:=\frac{4}{3} \cdot \frac{\mathrm{R}_{1}}{\mathrm{~A}_{\text {pin }}} \quad{ }^{\tau_{1}}=6602.95 \mathrm{psi} \\
& \mathrm{\tau}_{2}:=\frac{4}{3} \cdot \frac{\mathrm{R}_{2}}{\mathrm{~A}_{\text {pin }}} \quad{ }^{\tau_{2}}=-3677.39 \mathrm{psi} \\
& \mathrm{\tau}_{3}:=\frac{4}{3} \cdot \frac{\mathrm{R}_{3}}{\mathrm{~A}_{\text {pin }}} \quad{ }^{\tau_{3}}=-2900.75 \mathrm{psi}
\end{aligned}
$$


Subject: 22 Ton Steel Waste Disposal Box Lifting Bail Design

Preparer: BD Flanagan $\mathrm{ZPOF}$

Page: 11 of $\underline{20}$

Checker: DL Riley

Qeebfor

$$
\begin{aligned}
& \tau_{4}:=\frac{4}{3} \cdot \frac{\mathrm{R}_{4}}{\mathrm{~A}_{\text {pin }}} \quad{ }^{4} 4=-2581.74 \mathrm{psi} \\
& i_{5}:=\frac{4}{3} \cdot \frac{\mathrm{R}_{5}}{\mathrm{~A}_{\text {pin }}} \quad i_{5}=-1567.17 \mathrm{psi} \\
& \tau_{6}:=\frac{4}{3} \cdot \frac{R_{6}}{A_{\text {pin }}} \quad \tau_{6}=4124.1 \quad \mathrm{psi}
\end{aligned}
$$

Largest Shear Stress is at $t_{1}=6602.95 \mathrm{psi}$

Determine the bending stress at the reaction points:

$$
\begin{array}{ll}
c=0.44 \text { in } & I=0.0288 \mathrm{in}^{4} \\
\sigma_{1}:=\frac{M_{1} \cdot c}{I} & \sigma_{1}=0 \mathrm{psi} \\
\sigma_{2}:=\frac{M_{2} \cdot c}{I} & \sigma_{2}=8500.97 \mathrm{psi} \\
\sigma_{3}:=\frac{M_{3} \cdot c}{I} & \sigma_{3}=6654.36 \mathrm{psi} \\
\sigma_{4}:=\frac{M_{4} \cdot c}{I} & \sigma_{4}=6455.26 \mathrm{psi} \\
\sigma_{5}:=\frac{M_{5} \cdot c}{I} & \sigma_{5}=9032.29 \mathrm{psi} \\
\sigma_{6}:=\frac{M_{6} \cdot c}{I} & \sigma_{6}=-2354.23 \mathrm{psi}
\end{array}
$$

Largest bending stress at the reaction points is at $\sigma_{5}=9032.29$ psi 
Subject: 22 Ton Steel Waste Disposal Box Lifting Bail Design

Preparer: BD Flanagan Tax

Checker: DL Riley $L A B$

Page: 12 of 20

Date: $9 / 22 / 99$

Date: $9 / 22 / 99$

Determine the maximum principal stresses at the reaction points:

$$
\begin{aligned}
& \sigma_{\mathrm{p} 1}:=\frac{\sigma_{1}}{2}+\left[\left(\frac{\sigma_{1}}{2}\right)^{2}+\tau_{1}\right]^{.5} \quad \sigma_{\mathrm{pl}}=6602.95 \mathrm{psi} \\
& \left.\sigma_{\mathrm{p} 2}:=\frac{\sigma_{2}}{2}+\left[\left(\frac{\sigma_{2}}{2}\right)^{2}+r_{2}\right]^{2}\right]^{5} \quad \sigma_{\mathrm{p} 2}=9870.97 \mathrm{psi} \\
& \sigma_{\mathrm{p} 3}:=\frac{\sigma_{3}}{2}+\left[\left(\frac{\sigma_{3}}{2}\right)^{2}+\tau_{3}\right]^{2} \quad \sigma_{\mathrm{p} 3}=7741.3 \quad \text { psi } \\
& \sigma_{\mathrm{p} 4}:=\frac{\sigma_{4}}{2}+\left[\left(\frac{\sigma_{4}}{2}\right)^{2}+\tau_{4}^{2}\right]^{.5} \quad \sigma_{\mathrm{p} 4}=7360.79 \mathrm{psi} \\
& \left.\sigma_{\mathrm{p} 5}:=\frac{\sigma_{5}}{2}+\left[\left(\frac{\sigma_{5}}{2}\right)^{2}+\mathrm{i}_{5}\right]^{2}\right]^{5} \quad \sigma_{\mathrm{p} 5}=9296.48 \mathrm{psi} \\
& \sigma_{p 6}:=\frac{\sigma_{6}}{2}+\left[\left(\frac{\sigma_{6}}{2}\right)^{2}+\tau_{6}^{2}\right]^{.5} \quad \sigma_{p 6}=3111.68 \text { psi }
\end{aligned}
$$

Largest Principal Stress is $\quad \sigma_{\mathrm{p} 2}=9870.97 \mathrm{psi}$ 


\section{ENGINEERING SAFETY EVALUATION}

Subject: 22 Ton Steel Waste Disposal Box Lifting Bail Design

Preparer: BD Flanagan

Page: 13 of 20

Checker: DL Riley

Determine Maximum Principal Shear Stress:

$$
\begin{aligned}
& { }^{\mathrm{p} p 1}:=\left[\left(\frac{\sigma_{1}}{2}\right)^{2}+\tau_{1}{ }^{2}\right]^{.5} \quad \tau_{\mathrm{pl}}=6602.95 \mathrm{psi} \\
& { }^{\tau} \mathrm{p} 2:=\left[\left(\frac{\mathrm{\sigma}_{2}}{2}\right)^{2}+\tau_{2}{ }^{2}\right]^{.5} \quad \tau_{\mathrm{p} 2}=5620.48 \mathrm{psi} \\
& { }^{\tau} \mathrm{p}_{3}:=\left[\left(\frac{\sigma_{3}}{2}\right)^{2}+\tau_{3}\right]^{]^{5}} \quad{ }^{\tau} \mathrm{p}=4414.12 \text { psi } \\
& \left.\tau_{\mathrm{p} 4}:=\left[\left(\frac{\sigma_{4}}{2}\right)^{2}+\tau_{4}\right]^{2}\right]^{5} \quad{ }^{t} \mathrm{p} 4=4133.16 \mathrm{psi} \\
& { }^{\tau} \mathrm{p} 5:=\left[\left(\frac{\sigma_{5}}{2}\right)^{2}+\tau_{5}\right]^{]^{5}} \quad \tau_{\mathrm{p} 5}=4780.33 \mathrm{psi} \\
& { }^{\tau} \mathrm{p}_{6}:=\left[\left(\frac{{ }_{0} 6}{2}\right)^{2}+\tau_{6}^{2}\right]^{.5} \quad{ }^{\mathrm{T}} \mathrm{p} 6=4288.8 \mathrm{psi}
\end{aligned}
$$

Largest Principal Shear Stress is ${ }^{\tau} \mathrm{p} 1=6602.95 \mathrm{psi}$ 


\section{ENGINEERING SAFETY EVALUATION}

Subject: 22 Ton Steel Waste Disposal Box Lifting Bail Design

Page: 14 of 20

Preparer:

Checker:
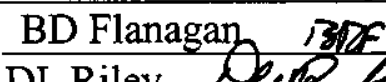

Date: $9 / 22 / 99$

Date: $9 / 22 / 99$

Determine Bending Stresses from maximum bending moments between reaction points:

Maximum moment is: $M_{\max 4}=-2151.02$ in ${ }^{*} \mathrm{bt}$

$$
\begin{aligned}
& \mathrm{c}:=.5 \cdot \frac{7}{8} \quad \mathrm{I}:=\frac{\pi}{4} \cdot(\mathrm{c})^{4} \\
& \sigma_{\mathrm{b}}:=\left|\mathrm{M}_{\max 4}\right| \frac{\mathrm{c}}{\mathrm{I}} \\
& \sigma_{\mathrm{b}}=32705.52 \quad \mathrm{psi}
\end{aligned}
$$

Determine Bearing Stress on pin:

Area length of teeth on hinge $L:=7.0$ in

Diameter of pin $\quad D_{p}:=.875$ in

Bearing stress $\quad \sigma_{b r}:=\frac{F}{L \cdot D_{p}} \quad \sigma_{b r}=4897.96 \quad$ psi

Pin Allowable Stresses:

AISI 41L40 - 120,000 psi yield strength

Allowable including a 3:1 Factor of Safety for lifting

$$
\sigma_{\text {all }}:=\frac{1}{3} \cdot 120000 \quad \sigma_{\text {all }}=40000 \mathrm{psi}
$$

Shear allowable using maximum shear stress theory predicts yield stress for shear at $1 / 2 \sigma_{\text {all }}$ (Shigley, 1989)

$$
{ }^{\tau} \text { all }:=5 \cdot \sigma_{\text {all }} \quad \tau_{\text {all }}=20000 \mathrm{psi}
$$




\section{ENGINEERING SAFETY EVALUATION}

Subject: 22 Ton Steel Waste Disposal Box Lifting Bail Design

Preparer: BD Flanagan I T.F

Checker: DL Riley DêB fo

Page: 15 of $\underline{20}$

Date: $\overline{9 / 22} / 99$

Date: $9 / 22 / 99$

Compare maximum stresses to allowables:

Largest shear stress at reaction points

$$
\tau_{1}=6602.95 \text { psi } \quad \text { MS }_{\mathrm{a}}:=\frac{{ }^{\tau} \text { all }}{\tau_{1}}-1 \quad \text { MS }_{\mathrm{a}}=2.03
$$

Largest bending stress at reaction points and margin of safety

$$
{ }^{\sigma_{5}}=9032.29 \mathrm{psi} \quad \mathrm{MS}_{\mathrm{b}}:=\frac{{ }^{\sigma} \text { all }}{\sigma_{5}}-1 \quad \mathrm{MS}_{\mathrm{b}}=3.43
$$

Largest principal stresses at reaction points and margin of safety

$$
\sigma_{\mathrm{p} 2}=9870.97 \mathrm{psi} \quad \mathrm{MS}_{\mathrm{c}}:=\frac{{ }^{\sigma} \text { all }}{\sigma_{\mathrm{p} 2}}-1 \quad \mathrm{MS}_{\mathrm{c}}=3.05
$$

Largest principal shear stress at reaction points and margin of safety

$$
\tau_{\mathrm{pl}}=6602.95 \text { psi } \quad \mathrm{MS}_{\mathrm{d}}:=\frac{{ }^{\tau} \text { all }}{\tau^{\tau} \mathrm{p} 1}-1 \quad \mathrm{MS}_{\mathrm{d}}=0.22
$$

Largest bending stress between reaction points and margin of safety

$$
{ }^{\sigma_{b}}=32705.52 \text { psi } \quad \mathrm{MS}_{\mathrm{d}}:=\frac{\sigma_{\text {all }}}{\sigma_{\mathrm{b}}}-1 \quad \mathrm{MS}_{\mathrm{d}}=0.22
$$

Bearing stress on pin and margin of safety

$$
\sigma_{b r}=4897.96 \text { psi } \quad M S_{e}:=\frac{{ }^{\tau} \text { all }}{\sigma_{b r}}-1 \quad M S_{e}=3.08
$$




\section{ENGINEERING SAFETY EVALUATION}

Subject: 22 Ton Steel Waste Disposal Box Lifting Bail Design

Preparer: BD Flanagan

Checker: DL Riley

Page: 16 of $\underline{20}$

Date: $\overline{9 / 22} / 99$

Date: $9 / 22 / 99$

\section{Evaluation of Lifting Bails}

Both top and bottom lifting bails have the same total length of teeth of $7.0 \mathrm{in}$. Therefore, stresses will be equal. Loading for each bail is $30,000 \mathrm{lbf}$.

Width of bail:

Total length of teeth:

Diameter of shaft hole:

Length of shear tear-out path:

$$
L_{\mathrm{S}}:=\left(.90 \cdot \text { in }-\frac{\phi h}{2}\right)+\left(1.0 \cdot \mathrm{in}-\frac{\phi \mathrm{h}}{2}\right) \quad \mathrm{L}_{\mathrm{s}}=1.02 \text { ・in }
$$

Minimum tensile area:

$$
A_{t}:=L \cdot(W-\phi h)
$$

$$
\mathrm{A}_{t}=7.85 \cdot \mathrm{in}^{2}
$$

Shear tear-out area:

$$
A_{s}:=L_{s} \cdot L
$$

$$
A_{s}=7.15 \cdot \mathrm{in}^{2}
$$

Bearing area:

$$
A_{b}:=L \cdot \phi h
$$

$$
A_{b}=6.15 \cdot \text { in }^{2}
$$

Tensile Stress:

$$
\sigma_{t}:=\frac{F}{A_{t}} \quad \sigma_{t}=3819.7 \cdot p s i
$$

Shear Tear-Out Stress: $\quad{ }^{i} \mathrm{t}:=\frac{F}{\mathrm{~A}_{\mathrm{S}}} \quad{ }^{\mathrm{t}} \mathrm{t}=4193.45$ 。psi

Bearing Stress:

$$
\sigma_{\mathrm{bs}}:=\frac{F}{\mathrm{~A}_{\mathrm{b}}} \quad \sigma_{\mathrm{bs}}=4881.22 \cdot \mathrm{psi}
$$


Subject: 22 Ton Steel Waste Disposal Box Lifting Bail Design

Preparer: BD Flanagan

Checker: DL Riley

Page: 17 of 20

Date: $9 / 22 / 99$

Date: $9 / 22 / 99$

Lifting Bail Allowable Stresses:

AISI 1040 - 42,000 psi yield strength

Allowable including a 3:1 Factor of Safety for lifting

$$
\text { of } \text { all }:=\frac{1}{3} \cdot 42000 \cdot \mathrm{psi} \quad \mathrm{ot}_{\mathrm{all}}=14000 \cdot \mathrm{psi}
$$

Shear allowable using maximum shear stress theory predicts yield stress for shear at $1 / 2 \sigma b_{\text {all }}$ (Shigley, 1989)

$$
\mathrm{tb}_{\mathrm{all}}:=.5 \circ \mathrm{b}_{\mathrm{all}} \quad \mathrm{tb} \mathrm{all}_{1}=7000 \circ \mathrm{psi}
$$

Compare maximum stresses to allowables:

Tensile stress and margin of safety

$$
\sigma_{t}=3819.7 \cdot p s i \quad M S_{f}:=\frac{\sigma_{a} \text { all }}{\sigma_{t}}-1 \quad M S_{f}=2.67
$$

Shear tear-out stress and margin of safety

$$
{ }^{t_{t}}=4193.45 \cdot p s i \quad \text { MS }_{\mathrm{g}}:=\frac{\tau^{\mathrm{t}} \text { all }}{\mathrm{t}_{\mathrm{t}}}-1 \quad \mathrm{MS}_{\mathrm{g}}=0.67
$$

Bearing stress and margin of safety

$$
\sigma_{b s}=4881.22 \text { opsi } \quad \mathrm{MS}_{\mathrm{h}}:=\frac{\sigma_{\text {all }}}{\sigma_{\mathrm{bs}}}-1 \quad \mathrm{MS}_{\mathrm{h}}=1.87
$$


Subject: 22 Ton Steel Waste Disposal Box Lifting Bail Design

Preparer: BD Flanagan

Checker: DL Riley

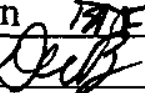

Page: 18 of 20

Date: $9 / 22 / 99$

Date: $9 / 22 / 99$

\section{Evaluation of Torsional Spring Force}

The torsional spring for this application was analyzed with spring information provided by Diamond Wire Spring Co. The spring has been designed by Diamond Wire Spring Co. to satisfy all of the requirements of the application. The material chosen is stainless steel. The model/part number assigned by Diamond Wire Spring Co. is $\phi 2.0 \times 3.86 \times \phi .374$ 17-7 (DWS 1999).

Angle between spring legs at the relaxed state: $\theta=55 \mathrm{deg}$

Width of the spring from end to end:

$\mathrm{w}=3.86 \mathrm{in}$.

Number of turns in the spring:

$\mathrm{n}=9.35$

Diameter of the spring wire used:

$\mathrm{d}=.37 \mathrm{in}$.

Outer diameter of the spring:

$\mathrm{D}=2.00 \mathrm{in}$

Volume of top lifting bail (derived by AutoCAD): $\quad \mathrm{V}:=404.47 \cdot \mathrm{in}^{3}$

Density of steel (Shigley, 1989): $\quad:=.283 \cdot \frac{\mathrm{lb}}{\mathrm{in}^{3}}$

Weight of top lifting bail: $\quad W:=\rho \cdot V \quad W=114.06 \mathrm{lb}$

Centroid location (derived by AutoCAD): $Y_{\text {bail }}=7.21$ in

Maximum torque to overcome (at $0 \mathrm{deg})$ :

$$
T_{0}:=W \cdot Y_{\text {bail }} \cos (0 \cdot \mathrm{deg}) \quad T_{0}=825.29 \cdot \mathrm{inlb}
$$

Torque at 45 deg:

$$
\mathrm{T}_{45}:=\mathrm{W} \cdot \mathrm{Y}_{\text {bail }} \cos (45 \cdot \mathrm{deg}) \quad \mathrm{T}_{45}=583.57 \cdot \mathrm{in} \mathrm{lb}
$$

Torque at 60 deg:

$$
\mathrm{T}_{60}:=\mathrm{W} \cdot \mathrm{Y}_{\text {bail }} \cos (60 \cdot \mathrm{deg}) \quad \mathrm{T}_{60}=412.65 \text { in } \mathrm{lb}
$$

WMNW.0D1 3/99 
Subject: 22 Ton Steel Waste Disposal Box Lifting Bail Design

Preparer: BD Flanagan

Page: 19 of 20

Checker:

DL Riley

Determine the steady state angle of the lifting bail.

Spring rate $S_{r}:=9.7067 \cdot \frac{\text { inlb }}{\mathrm{deg}}$

Weight of top lifting bail $\mathrm{W}=114.47 \mathrm{lb}$.

Centroid location bail $Y_{\text {bail }}=7.21$ in

Steady state angle

$$
\begin{aligned}
& \mathrm{T}_{\mathrm{a}}:=\mathrm{S}_{\mathrm{r} \cdot \phi \cdot d e g} \\
& \mathrm{~T}_{\mathrm{b}}:=\mathrm{W} \cdot \mathrm{Y}_{\text {bail }} \cos (\phi \cdot \mathrm{deg}) \\
& \mathrm{T}_{\mathrm{a}}:=\mathrm{T}_{\mathrm{b}} \\
& \phi:=52.16
\end{aligned}
$$


HNF-5409, Rev. 0

\section{ENGINEERING SAFETY EVALUATION}

Subject: 22 Ton Steel Waste Disposal Box Lifting Bail Design

Preparer: BD Flanagan BRF

Checker: DL Riley $\angle H 13$ /

Page: 20 of 20

Date: $\overline{9 / 22 / 99}$

Date: $9 / 22 / 99$

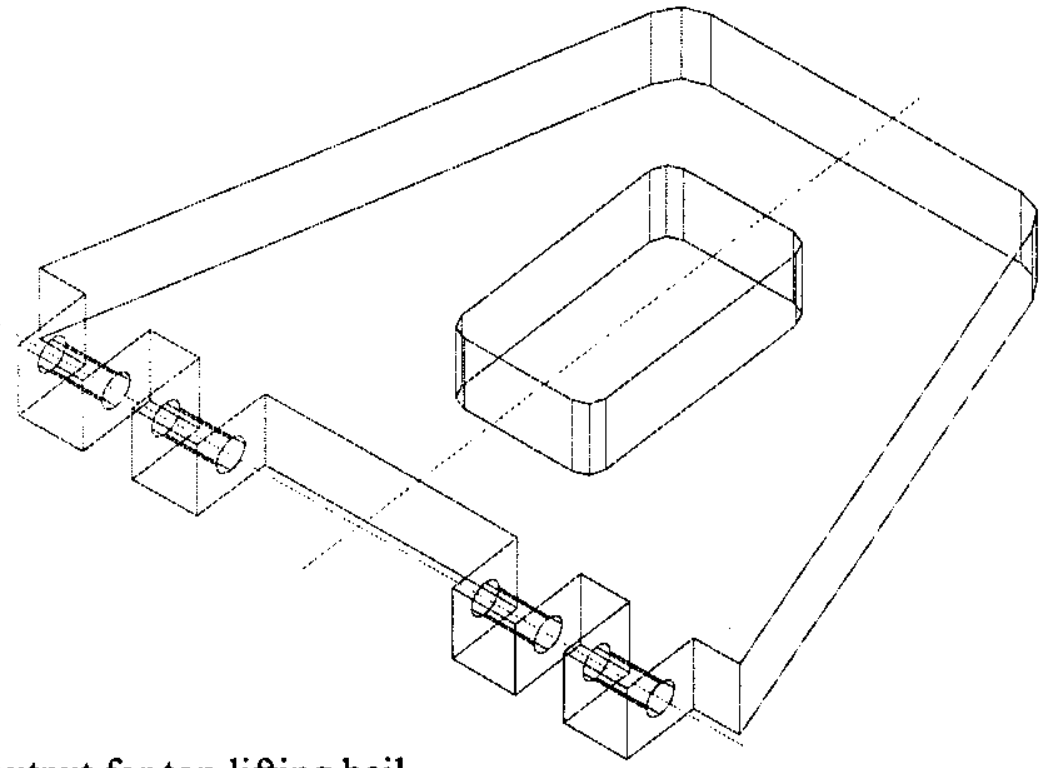

*AutoCAD output for top lifting bail

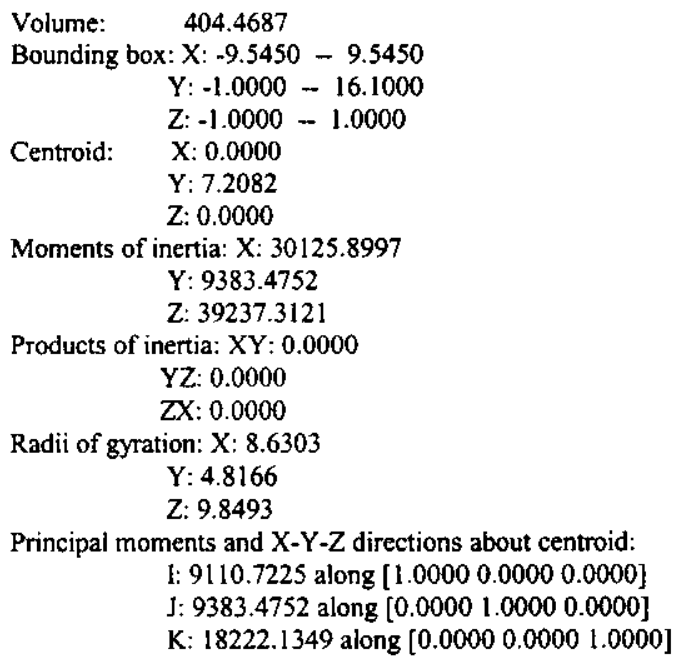

Principal moments and $X-Y-Z$ directions about centroid: 1: 9110.7225 along [ 1.00000 .00000 .0000$]$ $\mathrm{J}: 9383.4752$ along $[0.00001 .00000 .0000]$

$\mathrm{K}: 18222.1349$ along $[0.00000 .00001 .0000]$

*AutoCAD is a trademark of AutoDesk, Inc. WMNW.001 3/99 